\title{
Interactive comment on "Uncertainty of gridded precipitation and temperature reference datasets in climate change impact studies" by Mostafa Tarek et al.
}

\section{Anonymous Referee \#2}

Received and published: 23 December 2020

The manuscript by Tarek et al compares nine precipitation and two temperature datasets for 1145 African catchments to understand the uncertainties they introduce when used in hydrological modelling applications. The study is substantial, addresses a significant community challenge, and offers practical guidance to others in the field. On that basis I judge that it will be of clear interest to the readership of HESS, with substantial impact in Africa and insights applicable more widely. There are three major points which the authors should address in revision, and some minor points which they might wish to consider too: 
1. Bias correction is applied but only five lines are given to the description of the method used. It is essential to have more information on the bias corrections made, especially at rarely seen precipitation quantiles, given the sparsity of data in this region.

2. Hydrological modelling uncertainty (L240ff): more information is needed to attribute uncertainty in the hydrological modelling part of the work. It is unclear at present which components of the hydrological models contribute greatest uncertainty, especially with the HMETS model.

3. Applicabilty (L360 onwards): one of the key virtues claimed in the introduction is the guidance offered to future researchers tackling this problem afresh. Yet in this section the reader is left with the sense that there is no clear advantage to any particular dataset, with conclusions left as context dependent. Clearly that's a valid finding but it needs to be reflected in the stance that the paper offers in its opening and concluding paragraphs.

Minor comments / editorial points

Reference formatting is inconsistent with many additional brackets, etc. L173: hypodermic -> subsurface Figure 5: annotations and labels are hard to read; please enlarge

Interactive comment on Hydrol. Earth Syst. Sci. Discuss., https://doi.org/10.5194/hess-2020$517,2020$. 